Int. J. Odontostomat.,

14(3):393-399, 2020.

\title{
Evaluación del Índice de Korkhaus en Adultos Chilenos con Oclusión Normal
}

\author{
Evaluation of Korkhaus Index in Chilean Adult Individuals with Normal Occlusion
}

\author{
Nicolas Schleyer Daza' \& Francisca Couve Pérez²
}

SCHLEYER, D. N. \& COUVE, P. F. Evaluación del índice de Korkhaus en adultos chilenos con oclusión normal. Int. J. Odontostomat., 14(3):393-399, 2020.

RESUMEN: El examen de modelos es una de las múltiples herramientas diagnósticas que posee la ortodoncia y ortopedia dentomáxilofacial. Los valores con que se construyó su norma estadística están basados en poblaciones específicas y no reflejan las diferencias morfológicas existentes en el complejo cráneofacial entre las distintas etnias. El propósito de esta investigación fue identificar las longitudes transversales y sagitales de los maxilares de la población adulta mestiza chilena con oclusión normal y compararlas con el índice de Korkhaus basado en una población adulta caucásica alemana. Se reclutaron 20 estudiantes de pregrado de la Facultad de Odontología de la Universidad de Valparaíso con oclusión normal. Se registraron ambas arcadas dentarias con yeso y se aplicó el índice de Korkhaus: se midió la longitud interpremolar, la longitud intermolar, la longitud anterior superior y la longitud anterior inferior. La comparación entre los valores de la muestra y los valores referenciales del índice de Korkhaus fue hecha con la prueba de rangos de Wilcoxon mediante el software estadístico STATA 15.0 y se consideraron significativos valores de $p<0,05$. Se encontró una diferencia estadísticamente significativa en el valor de la longitud anterior superior ( $p-0,013)$; para la longitud interpremolar ( $p-0,9552)$ la longitud intermolar $(p-0,8223)$ y la longitud anterior inferior ( $p-0,0452)$ no se hallaron diferencias. La mayor magnitud de la longitud anterior superior de la arcada dentaria respecto a la población adulta caucásica alemana se debe al rasgo de protrusión dentaria presente en la población mestiza chilena, lo cual no altera las características de una oclusión normal.

PALABRAS CLAVE: arcada dentaria, modelo dental, raza caucásica, Chile.

\section{INTRODUCCIÓN}

La complejidad de las características de crecimiento y desarrollo de cada individuo ha hecho que el diagnóstico en ortodoncia y ortopedia dentomáxilofacial tenga un papel preponderante desde sus inicios. Bien documentadas son las diferencias morfológicas que existen en el complejo cráneofacial desde el tamaño, morfología coronaria y radicular de los dientes hasta el tamaño de los huesos maxilares, las cuales varían según el sexo (Scott \& Turner II, 1988), el estilo de vida y la dieta (Lavelle, 1972).

Para precisar el diagnóstico, se han creado numerosas herramientas; una de ellas es el estudio de modelos, el cual nos permite observar detalladamente ambas arcadas dentarias en todos los planos del espacio sin la presencia de los tejidos blandos, con el fin de realizar mediciones intra e interarcada en sentido transversal, sagital y vertical (Cuoto \& Nice, 2006). Arcadas dentarias congruentes y de tamaño óptimo se correlacionan con un correcto overbite y overjet, contactos oclusales estables, sin interferencias unido a una óptima salud periodontal y articular (Smith et al., 2000).

Pont (1909) fue uno de los primeros autores en relacionar el tamaño de los dientes con la longitud del arco dentario; creó un índice basado en población francesa con oclusión normal, el cual relacionaba la longitud transversal de las arcadas dentarias con el tamaño de los incisivos anterosuperiores (Walter, 1953). En 1939, Gustav Korkhaus modificó el índice de Pont al aplicarlo en población alemana, agregando la longitud sagital (Cuoto \& Nice).

Los estudios que utilizan el índice de Korkhaus son escasos. Aún así, al comparar la longitud transversal de las arcadas en población no caucásica es posi-

\footnotetext{
${ }^{1}$ Programa de Especialización en Ortodoncia y Ortopedia Dentomáxilofacial, Facultad de Odontología, Universidad de Valparaíso, Valparaíso, Chile. 2 Ortodoncista, Profesora Asociada Facultad de Odontología, Universidad de Valparaíso, Valparaíso, Chile.
} 
ble observar diferencias: en población africana (Lombardo et al., 2015) y coreana (Park et al., 2017) se observan arcos dentarios más amplios; en Malasia, arcos más estrechos (Purmal et al., 2013); y, al comparar las medidas de los arcos en diferentes ascendencias poblacionales de un mismo país existen diferencias entre sí (Bedoya-Rodríguez et al., 2016; Alkadhi et al., 2018). Los reducidos estudios en población chilena no están aplicados en dentición permanente; se enfocan en la comparación del ancho intercanino e intermolar en dentición mixta (Vargas et al., 2013) o caracterizar la forma de las arcadas de las poblaciones autóctonas (Saavedra et al., 1987).

El impacto de una herramienta diagnóstica no validada en una población específica incide en la generación de sub o sobretratamientos, afectando el correcto funcionamiento del sistema estomatognático, la estética facial (Taghavi Bayat et al., 2017) y comprometiendo la estabilidad del resultado del tratamiento (Edman Tynelius et al., 2015).

El objetivo de este estudio fue determinar las dimensiones de los anchos transversales y sagitales de los maxilares de una muestra de individuos de población mestiza chilena con oclusión normal y crecimiento terminado de la comuna de Valparaíso, V Región, Chile y compararlas con el índice de Korkhaus basado en una población caucásica alemana.

\section{MATERIAL Y MÉTODO}

Se realizó un estudio de tipo transversal sobre modelos de estudio de alumnos que cursaron el pregrado en la Facultad de Odontología de la Universidad de Valparaíso entre los años 2013 y 2018. El registro de los modelos de estudios se obtuvo previa aprobación de la investigación por parte del comité de ética de la Facultad de Odontología de la Universidad de Valparaíso y la firma del consentimiento informado de los alumnos seleccionados

Se aplicó una encuesta de reclutamiento para descartar a individuos con antecedentes de tratamiento de ortopedia y/u ortodoncia, extracciones de dientes permanentes (sin considerar los terceros molares), persistencia de diente temporal o agenesia de diente permanente, rehabilitación en base a prótesis fija y padres extranjeros. Los sujetos que cumplieron con los requisitos de preselección fueron examinados clínicamente aplicando los criterios de inclusión y exclusión (Tabla I).

El cálculo de la muestra fue no probabilístico, por conveniencia, con un total de 20 sujetos que reunieron los requisitos de oclusión normal. Se tomaron registros de ambas arcadas dentarias con alginato (Tropicalgin, Zhermack®) y se realizó el vaciado con yeso piedra Elite $®$ blanco (Velmix $®)$. Para la medición de las distancias transversales de las arcadas dentarias se utilizó el set para diagnósticos según Korkhaus (Dentaurum®). Todas las acciones y mediciones fueron ejecutadas por el mismo investigador (NS).

Las variables analizadas, en la arcada superior, incluyeron el tamaño en milímetros $(\mathrm{mm})$ del ancho mesiodistal de los 4 incisivos, la longitud entre el centro del surco mesiodistal de ambos primeros premolares (longitud interpremolar superior, LIPS), la longitud entre la fosa central de ambos primeros molares (longitud intermolar superior, LIMS) y la longitud entre la LIPS al punto de contacto interproximal de los incisivos centrales proyectado en las caras vestibulares (longitud anterior superior, LAS). En la

Tabla I. Criterios de inclusión y exclusión para evaluar el índice de Korkhaus en adultos chilenos.

Criterios de inclusión

1. Dentición permanente.

2. Sin antecedentes de tratamiento de ortopedia ni ortodoncia.

3. Sin extracciones de dientes permanentes (no se consideran los terceros molares).

4. Neutroclusión molar y canina.

5. Overbite y Overjet de 2 a 2,5 milímetros.

7. Sin rotaciones ni diastemas.

Criterios de exclusión

1. Padres extranjeros.

2. Enfermedades genéticas o congénitas que alteren la forma y/o tamaño de los huesos maxilares.

3. Compresión maxilar.

4. Alteración del número, forma y/o tamaño de los dientes permanentes.

5. Persistencia de dientes temporales.

6. Tratamientos rehabilitadores que alteren el tamaño de los dientes permanentes. 
arcada inferior, se midió la longitud entre el punto de contacto vestibular de ambos primeros y segundos premolares (longitud interpremolar inferior, LIPI), la longitud entre la cúspide mediovestibular de ambos primeros molares (longitud intermolar inferior, LIMI) y la longitud entre la LIPI al punto de contacto interproximal de los incisivos centrales proyectado en las caras vestibulares (longitud anterior inferior, LAI) (Fig. 1).

Las mediciones registradas se evaluaron respecto a la relación de las magnitudes o ratio entre la suma incisiva superior y las longitudes transversales intrepremolar $(0,84)$ e intermolar $(0,65)$ y la longitud anterior superior $(0,625)$ del índice de Korkhaus. Para la determinación del valor de la longitud anterior inferior, se restaron $2 \mathrm{~mm}$ a la longitud anterior superior (Fig. 2) (Rakosi \& Jonas, 1992). La Tabla II ejemplifica las medidas esperadas al aplicar el índice a una determina suma incisiva superior.

\section{Longitud interpremolar $=\underline{\text { suma incisiva } \times 100}$ 84}

\section{Longitud intermolar $=\underline{\text { suma incisiva }} \times 100$ 65}

Longitud anterior de la arcada $=\underline{\text { suma incisiva } \times 100}$

Fig. 2. Fórmula del índice de Korkhaus. 160

El análisis estadístico lo realizó un experto de la Facultad de Odontología de la Universidad de Valparaíso con el software estadístico STATA 15.0. Dada la naturaleza no probabilística de los datos y lo acotado de la muestra, se utilizó estadística descriptiva con intervalos de confianza ajustados para mues- tras pequeñas, mientras que para el análisis inferencial se ocuparon análisis no paramétricos, los cuales no presentaban supuestos que cumplir. De esta forma, se describieron promedios, desviaciones estándar (D.E.), coeficientes de variación (C.V.) y percentiles (p) 25, 50 (mediana) y 75 para los valores de la muestra. Para la comparación de medias se usó la prueba de rangos de Wilcoxon, tanto para comparar las diferencias de medianas absolutas como para los valores referenciales del índice Korkhaus y se consideraron significativos valores de $p<0,05$.

\section{RESULTADOS}

Los resultados en $\mathrm{mm}$. de las mediciones transversales y sagitales de las arcadas de los 20 individuos se detallan en la Tabla III.

Al analizar las medidas descriptivas de la muestra, se presentó un valor promedio de LIM con muy baja variabilidad (3\%). La mayor dispersión se observó para los valores LAS y LAI, donde alcanzaron un $7 \%$ y $8 \%$, respectivamente. Los valores LIP y LIM presentaron variabilidades menores a las teóricas, no así los valores LAS y LAI, los cuales fueron mayores (Tabla IV).

En la comparación de las diferencias entre los valores de la muestra y teóricas, se apreció que LIP fue 0,18 unidades mayor, LIM 0,11 unidades menor, LAS 0,71 unidades menor y LAI 0,64 unidades menor. Se registraron diferencias significativas entre las mediciones de LAS y LAI, ambos con $p<0,05$ (Tabla V).

En la Tabla VI se muestran las medidas descriptivas de las proporciones de la muestra y teóricas. Se observa que los promedios de LIP y LIM de la muestra se condicen con los factores multiplicativos propues-

Fig. 1. Puntos de referencias utilizados para medir las longitudes transversales y sagitales en ambas arcadas.
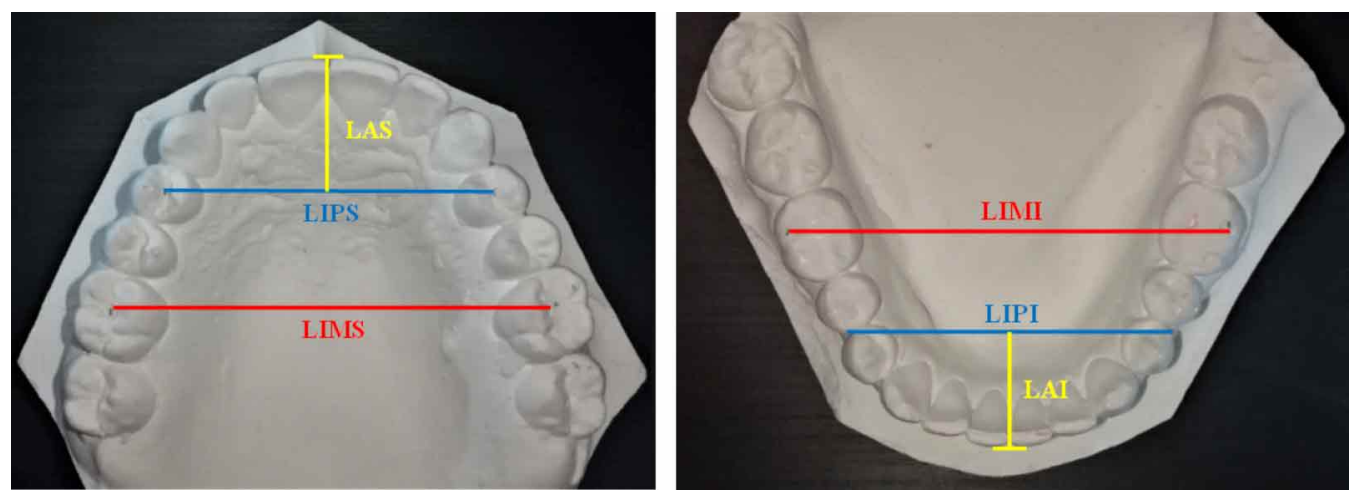
Tabla II. Extracto de los valores índice de Korkhaus al aplicar la fórmula. SI: suma incisiva superior; LIP: longitud interpremolar; LIM: longitud intermolar; LAS: longitud anterior superior; LAI: longitud anterior inferior.

\begin{tabular}{|c|c|c|c|c|}
\hline \multicolumn{5}{|c|}{ Índice de Korkhaus } \\
\hline SIS & LIP & LIM & LAS & LAI \\
\hline 29 & 34 & 44.5 & 17 & 15 \\
\hline 29.5 & 34.7 & 45.3 & 17.3 & 15.3 \\
\hline 30 & 35.5 & 46 & 17.5 & 15.5 \\
\hline 30.5 & 36 & 46.8 & 17.8 & 15.8 \\
\hline 31 & 36.5 & 47.5 & 18 & 16 \\
\hline 31.5 & 37 & 48.5 & 18.3 & 16.3 \\
\hline 32 & 37.5 & 49 & 18.5 & 16.5 \\
\hline 32.5 & 38.2 & 50 & 18.8 & 16.8 \\
\hline 33 & 39 & 51 & 19 & 17 \\
\hline 33.5 & 39.5 & 51.5 & 19.3 & 17.3 \\
\hline 34 & 40 & 52.5 & 19.5 & 17.5 \\
\hline 34.5 & 41 & 53 & 21.5 & 19.5 \\
\hline
\end{tabular}

tos por Korkhaus (84 y 65, respectivamente) pero no para LAS y LAI. Éstas últimas presentaron gran variabilidad (6\% y $7 \%$, respectivamente), de modo que los factores divisores en la muestra fueron 178 para LAS y 206 para LAI.

En términos de diferencias, sólo los valores de LAS y LAI de la muestra discreparon de los teóricos, siendo la primera de 8 y la segunda de 9 unidades porcentuales mayores. Para LAS, esta diferencia fue estadísticamente significativa, no así para el caso de LAI (Tabla VII).
Tabla III. Resultados en mm de las mediciones realizadas en la muestra. ILD: incisivo lateral derecho; ICD: incisivo central derecho; ICl: incisivo central izquierdo; ILI: incisivo lateral izquierdo; SIS: suma incisiva superior; LIP: longitud interpremolar; LIM: longitud intermolar; LAS: longitud anterior superior; LAI: longitud anterior inferior.

\begin{tabular}{|c|c|c|c|c|c|c|c|c|c|}
\hline \multirow[b]{2}{*}{ Muestra } & \multirow[b]{2}{*}{ ILD } & \multicolumn{7}{|c|}{ Resultados de la muestra } & \multirow[b]{2}{*}{ LAI } \\
\hline & & ICD & $\mathrm{ICl}$ & ILI & SIS & LIP & LIM & LAS & \\
\hline $\mathrm{A} 1$ & 7 & 8.5 & 8.5 & 7 & 31 & 38 & 49 & 17.5 & 15 \\
\hline B2 & 7 & 8.5 & 8.5 & 7 & 31 & 38 & 51 & 15.8 & 13.3 \\
\hline C3 & 7 & 9 & 9 & 7 & 32 & 38.3 & 49 & 16.5 & 14 \\
\hline D4 & 7 & 9 & 9 & 7 & 32 & 36 & 48 & 17.8 & 15.5 \\
\hline E5 & 7 & 8.5 & 8.5 & 7.5 & 31 & 37.8 & 49 & 17.8 & 15 \\
\hline $\mathrm{F} 6$ & 7 & 8 & 8 & 7 & 30 & 34.5 & 45 & 15 & 13 \\
\hline G7 & 8 & 9.5 & 9.5 & 8 & 35 & 41 & 50 & 18 & 15 \\
\hline $\mathrm{H} 8$ & 6.5 & 8 & 8 & 6.5 & 29 & 37.8 & 50 & 17.8 & 15.3 \\
\hline 19 & 7.5 & 9.5 & 9.5 & 8 & 34.5 & 37.8 & 47.3 & 20.3 & 18 \\
\hline J10 & 6.5 & 8 & 8 & 7 & 29.5 & 37.8 & 48.2 & 18 & 15.5 \\
\hline K11 & 8 & 9 & 9 & 7.5 & 33.5 & 38 & 47.5 & 19 & 16.3 \\
\hline L12 & 6.5 & 9 & 9 & 6.5 & 31 & 36 & 48.2 & 16.5 & 14.2 \\
\hline M13 & 7.5 & 9 & 9 & 7.5 & 33 & 37.5 & 48 & 18 & 16 \\
\hline N14 & 7 & 8.5 & 8.5 & 7 & 31 & 36 & 48 & 18.5 & 16 \\
\hline 015 & 7 & 9 & 9 & 7 & 32 & 37 & 51 & 18.5 & 16.5 \\
\hline P16 & 7 & 8.5 & 8.5 & 7 & 31 & 36 & 46 & 17 & 14.5 \\
\hline Q17 & 7 & 8.5 & 8.5 & 7 & 31 & 37 & 50.5 & 17 & 14.5 \\
\hline R18 & 7 & 9 & 9 & 6.5 & 31.5 & 39 & 49.2 & 18.2 & 15.8 \\
\hline S19 & 8 & 8.5 & 8.5 & 8 & 33 & 37.2 & 47.5 & 17.2 & 15 \\
\hline T20 & 7 & 9 & 9 & 7 & 32 & 39 & 48.5 & 18.7 & 16 \\
\hline
\end{tabular}

\begin{tabular}{lcclllll}
\hline & Variable & Promedio & D.E. & C.V. & p25 & p50 & p75 \\
\hline \multirow{3}{*}{ Valores } & SIS & 31,7 & 1,53 & 0,05 & 31 & 31,25 & 32,5 \\
de Ia & LIP & 37,48 & 1,4 & 0,04 & 36,5 & 37,8 & 38 \\
muestra & LIM & 48,55 & 1,54 & 0,03 & 47,75 & 48,35 & 49,6 \\
& LAS & 17,65 & 1,18 & 0,07 & 17 & 17,8 & 18,35 \\
& LAI & 15,22 & 1,15 & 0,08 & 14,5 & 15,15 & 16 \\
Valores & LIP & 37,3 & 1,8 & 0,05 & 36,5 & 36,75 & 38,25 \\
teóricos & LIM & 48,66 & 2,43 & 0,05 & 47,5 & 48 & 50 \\
& LAS & 18,36 & 0,77 & 0,04 & 18 & 18,15 & 18,75 \\
& LAI & 15,86 & 0,77 & 0,05 & 15,5 & 15,65 & 16,25 \\
\hline
\end{tabular}

Tabla IV. Medidas descriptivas del análisis del índice de Korkhaus de la muestra y teórico.
Tabla V. Diferencias de valores promedios entre mediciones del índice de Korkhaus de la muestra y teóricos junto a su resultado de test de signos de Wilcoxon.

\begin{tabular}{ccl}
\hline Variable & $\Delta$ & $P$-valor \\
\hline LIP & 0,18 & 0,7498 \\
LIM & $-0,11$ & 0,8812 \\
LAS & $-0,71$ & 0,0129 \\
LAI & $-0,64$ & 0,028 \\
\hline
\end{tabular}

Tabla VI. Medidas descriptivas de las proporciones de la muestra y teóricas de las mediciones para el índice de Korkhaus.

\begin{tabular}{cccccccc}
\hline & Variable & Promedio & D.E & CV & p25 & p50 & p75 \\
\hline \multirow{4}{*}{ Proporciones } & LIP & 0,84 & 0,04 & 0,05 & 0,81 & 0,86 & 0,87 \\
de la muestra & LIM & 0,65 & 0,04 & 0,06 & 0,63 & 0,64 & 0,68 \\
& LAS & 1,8 & 0,11 & 0,06 & 1,72 & 1,78 & 1,89 \\
& LAI & 2,09 & 0,15 & 0,07 & 1,96 & 2,06 & 2,19 \\
Proporciones & LIP & 0,84 & & & & & \\
teóricas & LIM & 0,65 & & & & & \\
\hline
\end{tabular}


En la Tabla VIII se aprecian los valores medios de cada uno de los parámetros del índice de Korkhaus; se observó una variabilidad muy pequeña, alrededor de un $1 \%$ para todas las mediciones, excepto para LAI donde fue un $2 \%$, lo cual refleja la precisión de los intervalos de confianza.

Finalmente, los factores del índice de Korkhaus medidos en la muestra mostraron valores de LIP y LIM iguales a los propuestos, mientras que los de LAS y LAI resultaron ser mayores (Tabla IX).

Tabla VII. Diferencias de valores promedios entre las proporciones del índice de Korkhaus de la muestra y teóricos junto a su resultado de test de signos de Wilcoxon.

\begin{tabular}{ccl}
\hline Variable & $\Delta$ & P-valor \\
\hline LIP & 0 & 0,9552 \\
LIM & 0 & 0,8223 \\
LAS & 0,08 & 0,013 \\
LAI & 0,09 & 0,0452 \\
\hline
\end{tabular}

Tabla VIII. Estimaciones de las mediciones del índice de Korkhaus de la muestra estudiada usando la técnica de Bootstrap con 100 repeticiones.

\begin{tabular}{lcccc}
\hline & Media & E.S. & \multicolumn{2}{c}{ IC 95 \% } \\
\hline SI & 31,7 & 0,33 & 31,05 & 32,35 \\
LIP & 37,49 & 0,26 & 36,97 & 38 \\
LIM & 48,55 & 0,32 & 47,91 & 49,18 \\
LAS & 17,66 & 0,23 & 17,2 & 18,11 \\
LAI & 15,22 & 0,23 & 14,77 & 15,67
\end{tabular}

Tabla IX. Estimaciones de las proporciones para el índice de Korkhaus de la muestra estudiada usando la técnica de Bootstrap con 100 repeticiones.

\begin{tabular}{ccccc}
\hline & Media & E.S. & \multicolumn{2}{c}{ IC 95 \% } \\
\hline LIP & 0,84 & 0,01 & 0,83 & 0,86 \\
LIM & 0,65 & 0,01 & 0,63 & 0,66 \\
LAS & 1,8 & 0,02 & 1,75 & 1,84 \\
LAI & 2,09 & 0,03 & 2,02 & 2,15 \\
\hline
\end{tabular}

\section{DISCUSIÓN}

En Chile, no existe información respecto a la prevalencia de una oclusión normal natural en la población. Sin embargo, es posible tener un panorama al analizar los datos de prevalencia de maloclusiones de diferentes investigaciones, aunque con una gran heterogeneidad de muestras y criterios de inclusión
(Espinoza et al., 2011). En el estudio más reciente hecho en la región de Valparaíso, y sólo considerando el apiñamiento como elemento de medición, existe un $84 \%$ de prevalencia entre los 12 y 15 años (Cueto et al., 2017). Lo anterior explica el reducido tamaño muestral con oclusión normal obtenido (20 individuos). Es importante recalcar que Korkhaus creó el índice sin informar el número de sujetos, el rango etario ni la conformación de la muestra por sexo.

No existe información en la literatura respecto a una comparación del índice de Korkhaus utilizando los mismos criterios de oclusión normal que se usaron para su construcción. Aunque reducidos, existen datos respecto a su precursor Pont: en Kuwait, las arcadas presentaron mayor tamaño en hombres que en mujeres (Rastegar-Lari et al., 2012); en Jordania, se hallaron arcadas dentarias estrechas en mujeres (AlOmari et al., 2007); y, en Australia (Dalidjan et al., 1995) y México (Carrizosa Celis et al., 2005) se observaron arcadas dentarias de menor longitud transversal en la población. Respecto a la situación en Chile, no se ha investigado el índice de Korkhaus. Si bien existen publicaciones con medición de los anchos intercaninos e intermolares, se han empleado índices de otros autores en dentición mixta (Vargas et al.) y con criterios de inclusión más flexibles al definir una oclusión normal (Ferrario et al., 1999). En resumen, ningún estudio ha mostrado similitud en los valores propuestos por algún índice de longitud de las arcadas dentarias entre las diferentes etnias.

En la presente investigación, el análisis estadístico del índice de Korkhaus entre una población alemana y una población mestiza chilena no arrojó diferencias estadísticamente significativas en los valores de la longitud interpremolar ( $p-0,9552)$, la longitud intermolar $(p-0,8223)$ y la longitud anterior inferior ( $p$ 0,0452 ). Esto demuestra la concordancia que existe entre los valores de ambas muestras, observándose arcadas dentarias bien proporcionadas respecto al tamaño dentario y sin protrusión ni retrusión del grupo incisivo inferior. Donde sí hubo una diferencia estadísticamente significativa fue en el valor de la longitud anterior superior ( $p-0,013)$, lo cual es compatible con una protrusión del grupo incisivo superior según Korkhaus. Este fenómeno puede explicarse por un aumento del tamaño vestíbulopalatino de uno o ambos incisivos centrales superiores o por un aumento del torque de estos mismos. Sin embargo, esta diferencia no se manifestó en un aumento del overjet o resalte que alterara los criterios de oclusión normal de esta investigación. 
Los estudios no arrojan diferencias en el tamaño vestíbulopalatino del incisivo central superior entre distintas poblaciones. Las diferencias han sido encontradas en el incisivo central inferior y los caninos superiores entre poblaciones de Nigeria y Gran Bretaña (Otuyemi \& Noar, 1996) y en caninos y molares de hombres en población egipcia, mexicana y estadounidense (Bishara et al., 1983). La comparación en una misma población muestra similar comportamiento, ya que se observa dimorfismo sexual entorno al canino superior, mostrando diferencias estadísticamente significativas respecto al tamaño del segundo premolar y primer molar superiores y del primer molar inferior (Axelsson \& Kirveskari, 1983). En población mestiza chilena, el valor del tamaño vestíbulopalatino del incisivo central es el que muestra el menor diámetro al ser comparado con el de población atacameña, pehuenche y mapuche (Saavedra et al.), por lo que el aumento de la longitud anterior superior de la arcada no es explicable por este motivo.

La otra alternativa a considerar en el aumento de la longitud anterior superior es la manifestación de un mayor torque de los incisivos centrales superiores. La biprotrusión dentaria es una característica esperable en arcadas dentarias estrechas. Sin embargo, es posible hallar individuos con oclusión normal sin afectación de ninguno de los parámetros que la caracterizan, incluso sin alteración del perfil y poseer un protrusión incisiva respecto a los valores cefalométricos establecidos por Ricketts (Montt et al., 2015).

En conclusión, el mayor torque en los incisivos centrales superiores es el responsable del aumento de la longitud anterior superior de la arcada dentaria en esta investigación al comparar una población caucásica alemana con una chilena mestiza. Es importante tener en consideración que el rasgo de protrusión dentaria es una característica de nuestra población y debe ponderarse, junto a otras herramientas diagnósticas, al momento de definir la terapéutica ortodóncica.

SCHLEYER, D. N. \& COUVE, P. F. Evaluation of Korkhaus index in Chilean adult individuals with normal occlusion. Int. J. Odontostomat., 14(3):393-399, 2020.

ABSTRACT:The model exam is one of many diagnostic tools in orthodontics and dentomaxillofacial orthopedics. The values on which the statistical norm were constructed, are based on specific populations and they do not reflect the morphological differences in the craniofacial complex between the different ethnic groups. The purpose of this study was to identify the transverse and sagittal maxillary and mandibular lengths of the Chilean mestizo adult population with normal occlusion, and compare them with the Korkhaus index based on a German Caucasian adult population. For this study 20 undergraduate students with normal occlusion were recruited from the Faculty of Dentistry of the University of Valparaíso. Both upper and lower dental casts were obtained from each student, and Korkhaus index was applied: the interpremolar length, intermolar length, upper anterior length and lower anterior length were measured. Comparison between the values of the sample and the reference values of the Korkhaus index was made with the Wilcoxon range test using the STATA 15.0 statistical software and significant values of $p<0.05$ were considered. A statistically significant difference was found in the value of the upper anterior length ( $p-0.013)$; for the interpremolar length $(p-0.9552)$ the intermolar length $(p-0.8223)$ and the lower anterior length $(p-0.0452)$ no differences were found. The greater magnitude of the dental arch upper anterior length compared to the German Caucasian adult population is due to dental protrusion present in the Chilean mestizo population, which does not affect the normal occlusion characteristics.

KEY WORDS: dental arch, dental model, Caucasian race, Chile.

\section{REFERENCIAS BIBLIOGRÁFICAS}

Al-Omari, I. K.; Duaibis, R. B. \& Al-Bitar, Z. B. Application of Pont's Index to a Jordanian population. Eur. J. Orthod., 29(6):62731, 2017.

Alkadhi, O. H.; Almahfouz, S. F.; Tokhtah, H. A. \& Binhuwaishel, L. A. Dental arch dimensions in Saudi adults. Int. J. Dent., 2018:2190250, 2018.

Axelsson, G. \& Kirveskari, P. Crown size of permanent teeth in Icelanders. Acta Odontol. Scand., 41(3):181-6, 1983.

Bedoya-Rodríguez, A.; Montoya-Gómez, J.; GonzálezBenavidez, V.; Tamayo-Cardona, J. A. \& Martínez-Cajas, C. $\mathrm{H}$. Forma y tamaño del arco dental en poblaciones de tres ascendencias étnicas colombianas. Rev. CES Odontol., 29(2):20-32, 2016.

Bishara, S. E.; Jakobsen, J. R.; Abdallah, E. M. \& Fernandez García, A. Comparisons of mesiodistal and buccolingual crown dimensions of the permanent teeth in three populations from Egypt, Mexico, and the United States. Am. J. Orthod. Dentofacial Orthop., 96(5):416-22, 1983.

Carrizosa Celis, L.; Ortiz Cruz, E.; Murrieta Pruneda, J. F. \& Juárez López, L. A. Estudio comparativo entre dos índices de predicción de la dimensión transversal de arcadas dentarias en mexicanos. Vertientes Rev. Espec. Cienc. Salud, 8(1-2):26-30, 2005.

Cueto, A.; Skog, F.; Muñoz, M.; Espinoza, S.; Muñoz, D. \& Martínez, D. Prevalencia de anomalías dentomaxilares y necesidad de tratamiento en adolescentes. Int. J. Odontostomat., 11(3):333-8, 2017.

Cuoto, R. \& Nice, L. Análisis de modelos: revisión de la literatura. Rev. Clin. Ortod. Dent., 5:64-76, 2006.

Dalidjan, M.; Sampson, W. \& Townsend, G. Prediction of dental arch development: an assessment of Pont's Index in three human populations. Am. J. Orthod. Dentofacial Orthop., 107(5):465-75, 1995. 
Edman Tynelius, G.; Petrén, S.; Bondemark. L. \& Lilja-Karlander, E. Five-year postretention outcomes of three retention methods--a randomized controlled trial. Eur. J. Orthod., 37(4):345-53, 2015.

Espinoza, A.; Parra, N.; Prieto, F.; Fernández, C. \& Venegas, V. Prevalencia de anomalías dentomaxilares y malos hábitos orales en pre-escolares de zonas rurales de la población beneficiaria del Servicio de Salud de Viña del Mar-Quillota. Rev. Chil. Ortod., 28(2):58-65, 2011.

Ferrario, V. F.; Sforza, C.; Colombo, A.; Carvajal, R.; Duncan, V. \& Palomino, $\mathrm{H}$. Dental arch size in healthy human permanent dentitions: ethnic differences as assessed by discriminant analysis. Int. J. Adult Orthodon. Orthognath. Surg., 14(2):15362, 1999.

Lavelle, C. L. Secular trends in different racial groups. Angle Orthod., 42(1):19-25, 1972.

Lombardo, L.; Coppola, P. \& Siciliani, G. Comparison of dental and alveolar arch forms between different ethnic groups. Int. Orthod., 13(4):462-88, 2015.

Montt, R. J.; Miquel, V. M. P. \& Oyonarte, W. R. Cephalometric characteristics in young individuals with normal occlusion and harmonic profiles in Chilean population. Int. J. Morphol., 33(1):237-44, 2015.

Otuyemi, O. D. \& Noar, J. H. A comparison of crown size dimensions of the permanent teeth in a Nigerian and a British population. Eur. J. Orthod., 18(6):623-8, 1996.

Park, S. J.; Leesungbok, R.; Song, J. W.; Chang, S. H.; Lee, S. W. \& Ahn, S. J. Analysis of dimensions and shapes of maxillary and mandibular dental arch in Korean young adults. J. Adv. Prosthodont., 9(5):321-7, 2017.

Purmal, K.; Alam, M. K.; Moganadass, D. D.; Zakariat, N. N. \& Cheong, N. W. The application and correlation of Pont's Index to the facial framework of three main ethnic groups in Malaysia. Aust. Orthod. J., 29(1):34-42, 2013.

Rakosi, T. \& Jonas, I. Análisis de Modelos. En: Rakosi, T. \& Jonas, I. (Eds.). Atlas de Ortopedia Maxilar: Diagnóstico. Barcelona, Ediciones Científicas y Técnicas S. A., 1992. pp.207-235.

Rastegar-Lari, T.; Al-Azemi, R.; Thalib, L. \& Ârtun, J. Dental arch dimensions of adolescent Kuwaitis with untreated ideal occlusion: variation and validity of proposed expansion indexes. Am. J. Orthod. Dentofacial Orthop., 142(5):635-44, 2012.

Saavedra, E.; Blanco, R. \& Cifuentes, L. Morfología máxilodentaria en poblaciones chilenas contemporáneas de distinto origen étnico. Odontol. Chil., 35:120-9, 1987.

Scott, G. R. \& Turner II, C. G. Dental anthropology. Ann. Rev. Anthropol., 17:99-126, 1988.

Smith, S. S.; Buschang, P. H. \& Watanabe, E. Interarch tooth size relationships of 3 populations: "does Bolton's analysis apply?". Am. J. Orthod. Dentofacial Orthop., 117(2):169-74, 2000.

Taghavi Bayat, J.; Huggare, J.; Mohlin, B. \& Akrami, N. Predicting orthodontic treatment need: reliability and validity of the Demand for Orthodontic Treatment Questionnaire. Eur. J. Orthod., 39(3):326-33, 2017.

Vargas, J.; Harnisch, A.; Torres, A.; Fierro, C. \& Pérez, A. Evaluación de anchos intercaninos e intermolares en escolares con dentición mixta, Comuna de Contulmo, Chile. J. Oral Res., 2(2):64-7, 2013

Walter, D. C. Changes in the form and dimensions of dental arches resulting from orthodontic treatment. Angle Orthod., 23(1):3-18, 1953.

\author{
Dirección para correspondencia: \\ Dr. Nicolas Schleyer \\ Residente del Programa de Especialización en Ortodoncia \\ y ODMF \\ Facultad de Odontología \\ Universidad de Valparaíso \\ Valparaíso \\ CHILE
}

Email: nico_schleyer@hotmail.com

Recibido: 16-12-2019

Aceptado: 23-02-2020 